\title{
Effects of Bak Foong Pill on Blood Coagulation and Platelet Aggregation
}

\author{
Yu Lin Gou, Alice Lok Sze Ho, Dewi Kenneth Rowlands, Yiu Wa Chung, and Hsiao Chang Chan* \\ Epithelial Cell Biology Research Center, Department of Physiology, The Chinese University of Hong Kong; N.T, Hong \\ Kong, P. R. China. Received September 20, 2002; accepted November 21, 2002
}

\begin{abstract}
The effects of Bak Foong Pill (BFP, also known as Bai Feng Wan), a preparation of crude drugs in wide clinical use for treatment of gynecological disorders, on blood coagulation and platelet aggregation were investigated. The anticoagulant effect of BFP was evaluated by using thrombin time (TT), prothrombin time (PT), and activated partial thromboplastin time (APTT) assays. Results showed that BFP 70\% ethanol extract (BFP-E-ext) significantly prolonged the $T T$ in a dose-dependent manner with values of 17.6, 38.3, and $50.4 \mathrm{~s}$ at concentrations of 4.0, 6.0, and $12.0 \mathrm{mg} / \mathrm{ml}$, respectively. Whereas, the BFP-E-ext did not show significant prolonging effect in PT and APTT assays. The results suggest that the anticoagulant effect of BFP is mediated by directly blocking thrombin, the key enzyme in the blood coagulation cascade. BFP-E-ext significantly inhibited platelet aggregation induced by collagen and adenosine diphosphate (ADP) with inhibition percentages of 74 and $52 \%$ at a concentration of $6.0 \mathrm{mg} / \mathrm{ml}$, respectively, whereas, it exhibited a weak inhibitory activity on platelet aggregation induced by archidonic acid (AA). Comparing to BFP-E-ext, the effects of BFP aqueous extract (BFP-W-ext) on both anticoagulant and antiplatelet activities were significantly less potent. Moreover, the effects of the 26 ingredients of BFP on blood coagulation and platelet aggregation were separately evaluated with 19 ingredient herbs exhibiting anticoagulant effect and 10 exhibiting antiplatelet effect. The anticoagulant and antiplatelet effects of BFP were collectively demonstrated by in vivo assays showing prolonged bleeding times after BFP treatment for two weeks. The results of the present studies may provide explanations for beneficial effects of BFP on the circulation and indicate its potential use for cardiovascular diseases.
\end{abstract}

Key words Bak Foong Pill; prothrombin time (PT); thrombin time (TT); activated partial thromboplastin time (APTT); platelet aggregation; anticoagulant

Blood coagulation is a host defense mechanism that assists in maintaining the integrity of the closed, high-pressure mammalian circulatory system after blood vessel injury. In the abnormal conditions, it is also involved in the thrombosis, atherosclerosis, inflammation and metastasis by the activation of enzymes in the coagulation cascade and the platelets. ${ }^{1)}$ The key enzyme, thrombin, and platelets, play an important role in the initiation of the coagulation process and involve in the formation of the fibrin clot and platelet plug in the vascular system. Thus, safe and effective inhibitors of thrombin and platelets should be useful tools in the treatment of venous thrombosis, arterial fibrillation, restenosis, arterial thrombosis, and in the prevention of myocardial infarction. Because of this, the modulation of thrombin by direct inhibitors and antiplatelet agents are widely sought goals in the development of anticoagulant agents. ${ }^{2)}$ A number of previous studies have tried to screened out potential candidates of anticoagulant and antiplatelet agents from plants, ${ }^{3,4)}$ and some herbs identified with potent anticoagulation and antiplatelet activities. $^{5,6)}$

Bak Foong Pill (BFP, also known as Bai Feng Wan), an over-the-counter traditional Chinese medicine with 26 ingredient herbs, has a long history for treating gynecological disorders, such as dysmenorrhea, irregular menstrual cycle and irregular bleeding. Clinical indications also suggest that BFP has beneficial effect on overall body functional systems including the cardiovascular, digestive system as well as the reproductive system. Our previous studies has demonstrated estrogenic effect of BFP and it beneficial effect on gastrointestinal secretion, ${ }^{7,8)}$ as well as preventing/delaying development of hypertension in spontaneous hypertension rats (Zhou et al. unpublished data). In order to evaluate the effect of BFP on the cardiovascular system, we undertook the present study to investigate its effect on blood coagulation and platelet aggregation both in vitro and in vivo using prothrombin time (PT), thrombin time (TT), activated partial thromboplastin time (APTT), and platelet aggregation assays. The same assays were also conducted to evaluate anticoagulant and antiplatelet activities of the 26 ingredients from BFP.

\section{MATERIALS AND METHODS}

Animals ICR mice and New Zealand white rabbits were obtained from the Laboratory Animal Service Center of the Chinese University of Hong Kong. Animals were maintained in an air-conditioned room with controlled temperature of $24 \pm 2{ }^{\circ} \mathrm{C}$ and humidity $55 \pm 15 \%$ in a $12 \mathrm{~h}$ light/dark cycle regulation. The animals were allowed food and water ad libitum. Animals were divided randomly into control and experimental groups.

Reagents Collagen, adenosine diphosphate (ADP), and archidonic acid (AA) reagents for platelet function testing were obtained from Chrono-Log Co. (Havertown, PA, U.S.A.). Thromborel ${ }^{\circledR} \mathrm{S}$ reagent, Dade ${ }^{\circledR}$ thrombin time reagent, and actin activated cephaloplastin reagent were purchased from Bade Behring Narburg GmbH (Marburg, Germany). Heparin sodium salt (from porcine intestinal mucosa, 187 USP units/mg), aspirin and indomethacin were obtained from Sigma Chemical Co. (St. Louis, MO, U.S.A.). All drugs were dissolved in Tris buffer ( $\mathrm{pH}$ 7.4) contained $0.9 \%$ sodium chloride. Other chemicals were of reagent grade and were from commercial sources.

Extraction of BFP and Ingredients of BFP Five hundred grams of Bak Foong Pills (obtained from Eu Yan Sang Company, Hong Kong) was grinded and passed the 80 meshes. The powder $(200 \mathrm{~g})$ of BFP was refluxed using $70 \%$ ethanol aqueous $(11 \times 3$ times). The filtrate was concentrated to 0.21 under $60^{\circ} \mathrm{C}$ and dried using a freeze dryer to afford 
the BFP-E-ext $(76 \mathrm{~g})$. The BFP powder $(100 \mathrm{~g})$ was extracted under $60^{\circ} \mathrm{C}$ by distilled water $(200 \mathrm{ml} \times 3$ times $)$ and dried by a freeze dryer to afford the BFP-W-ext $(30.5 \mathrm{~g})$. Of these 26 ingredients in the preparation of BFP were supplied by $\mathrm{Eu}$ Yan Sang Co. (Hong Kong). The each single powdered ingredient $(200 \mathrm{~g})$ was refluxed using $70 \%$ ethanol $(11 \times 3$ times). The filtrate was concentrated to 0.21 under $60^{\circ} \mathrm{C}$ and dried using a freeze dryer to afford the individual ingredient extract. All samples were stored at $0{ }^{\circ} \mathrm{C}$.

In Vitro Blood Clotting Assays ${ }^{11)}$ The plasma used for clotting assays was prepared by centrifuging citrated rabbit vine blood at a force of $1500 \times \boldsymbol{g}$ for at least $15 \mathrm{~min}$ under 10 ${ }^{\circ} \mathrm{C}$. The effects of extracts from Bak Foong Pills and ingredients (formulated in Bak Foong Pills) on coagulation of rabbit plasma in vitro were measured at $37^{\circ} \mathrm{C}$ on a coagulatemeter using PT, TT, and APTT assays. For PT assay, $150 \mu \mathrm{l}$ of $0.05 \mathrm{M}$ Tris buffer containing $0.1 \mathrm{M}$ sodium chloride $(\mathrm{pH} 7.5)$, $50 \mu \mathrm{l}$ of sample solution (extract dissolved in $0.05 \mathrm{M}$ Tris buffer), and $100 \mu \mathrm{l}$ citrated rabbit plasma were incubated for $3 \mathrm{~min}$ at $37^{\circ} \mathrm{C}$. Clotting was initiated by addition of $100 \mu \mathrm{l}$ of thromborel reagent (Thromborels was reconstituted in $4 \mathrm{ml}$ of distilled water). Similarly, the TT were measured by addition of $200 \mu \mathrm{l}$ thrombin reagent (Dade ${ }^{\circledR}$ thrombin reagent) to a mixture of rabbit plasma $100 \mu 1,0.05 \mathrm{M}$ Tris buffer $(\mathrm{pH} 7.5)$ $50 \mu \mathrm{l}$, and $50 \mu \mathrm{l}$ sample solution (extracts dissolved in Tris buffer), which were pre-incubated for $3 \mathrm{~min}$. The APTT was measured by incubating rabbit plasma $(100 \mu \mathrm{l}), 0.05 \mathrm{M}$ Tris buffer $(50 \mu \mathrm{l})$, and samples dissolved in Tris buffer $(50 \mu \mathrm{l})$ with the actin activated cephaloplastin reagent $(100 \mu \mathrm{l})$ for 3 min. Clotting was initiated by adding $25 \mathrm{~mm} \mathrm{CaCl}_{2}$ $(100 \mu \mathrm{l})$. Clotting times are based on the average of three separate determinations.

Bleeding Times ${ }^{12)}$ Bleeding times were measured as described previously. ${ }^{12)}$ Briefly, 2-3 months old ICR mice were treaded with BFP-E-ext $(2,4,6 \mathrm{~g} / \mathrm{kg}$, respectively, p.o.) or vehicle $(1 \mathrm{ml} / \mathrm{kg}$, p.o. $)$ for 2 weeks, followed by blotting from an amputated tail every $15 \mathrm{~s}$.

Platelet Aggregation Assays ${ }^{13)}$ Blood was collected from the rabbit ear vein and mixed with $3.8 \%$ sodium citrate at a ratio of $1: 9(\mathrm{v} / \mathrm{v})$. The blood was centrifuged at $190 \times \boldsymbol{g}$ for $10 \mathrm{~min}$ and the supernatant was obtained as the platelet rich plasma (PRP). The citrated blood was centrifuged at a force of $1500 \times \boldsymbol{g}$ for $15 \mathrm{~min}$ under $10^{\circ} \mathrm{C}$ to afford the platelet poor plasma (PPP). The PRP was adjusted platelets to $3.8 \times$ $10^{8}$ platelets $/ \mathrm{ml}$. Platelet aggregation was measured by a turbidimetric method using a Whole Blood Lumi-Aggregometer (Chrono-Log Corp., Havertown, PA, U.S.A.). All glassware was siliconized. The platelet rich plasma (PRP, $300 \mu \mathrm{l}$ ) was incubated with $30 \mu \mathrm{l}$ samples (extract dissolved in Tris buffer) at $37^{\circ} \mathrm{C}$ for $3 \mathrm{~min}$, after which the inducers such as collagen $(4 \mu \mathrm{g} / \mathrm{ml}), \operatorname{ADP}(15 \mu \mathrm{M})$, and AA $(7.5 \mathrm{~mm})$ were added to initiate aggregation. The percentage of aggregation was determined in $10 \mathrm{~min}$. The absorbance of the PRP suspension was taken as $0 \%$ aggregation, and the absorbance of the PPP was taken as $100 \%$ aggregation. All experiments were measured in triplicate.

Data Analysis Results were expressed as mean \pm S.D. The Student's $t$-test was employed to analyses experimental data. A " $p$ " value of less then 0.05 was considered statistically significant.

\section{RESULTS}

To assess the activities of BFP on anticoagulation and antiplatelet aggregation, we examined effects of BFP both in $70 \%$ ethanol and aqueous extracts, BFP-E-ext and BFP-Wext, as well as its 26 ingredients (ethanol extracts) on blood coagulation and platelet aggregation functions.

Effects of BFP on Blood Coagulation PT, TT, and APTT assays were used to evaluate the effect of BFP on blood coagulation. As shown in Fig. 1, the BFP-E-ext significantly prolonged the TT $(p<0.001)$ in a dose-dependent manner, with the values of $17.6 \pm 0.2,38.3 \pm 0.4$, and $50.4 \pm 1.3$ (s) at dosages of $4.0,6.0$, and $12.0 \mathrm{mg} / \mathrm{ml}$, respectively. The BFP-W-ext showed less potent effect on prolonging TT $(p<0.001)$ with values of $23.2 \pm 0.2$ and $29.1 \pm 0.8(\mathrm{~s})$ at higher concentrations of 12.0 and $20.0 \mathrm{mg} / \mathrm{ml}$, respectively. However, both BFP-E-ext and BFP-W-ext did not produce significant effect on prolonging clotting activities in PT (Fig. $2)$ and APTT assays $(22.1 \pm 0.6$ and $18.9 \pm 0.6 \mathrm{~s}$. at concentrations of 4.0 and $12.0 \mathrm{mg} / \mathrm{ml})$.

In order to demonstrate anticoagulant components of BFP, we examined the effects of the individual ingredient of BFP on prolongation of clotting times using PT, TT, and APTT assays at a concentration of $2.0 \mathrm{mg} / \mathrm{ml}$. As shown in Table 1, of the 26 ingredients of BFP, 17 ingredients showed signifi-

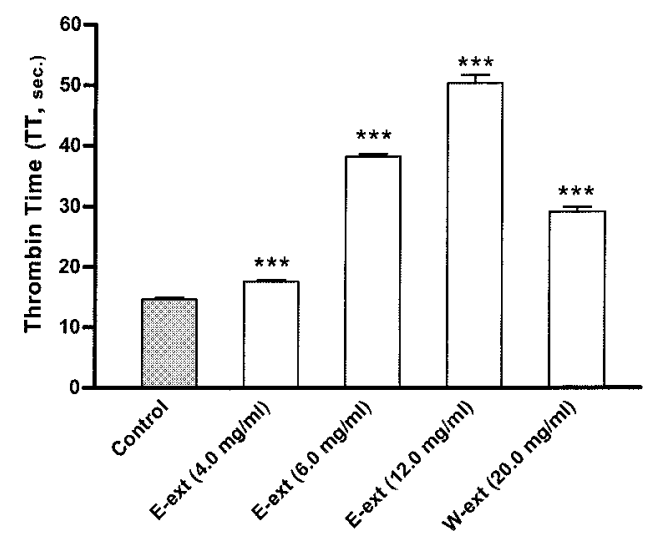

Fig. 1. Effects of BFP on TT Clotting Assay

Each bar is reported as mean (s) \pm S.D. $(n=3-5) ; * * * p<0.001$ significantly different from control, Student's $t$-test. E-ext. and W-ext. mean the BFP $70 \%$ ethanol and aqueous extracts respectively.

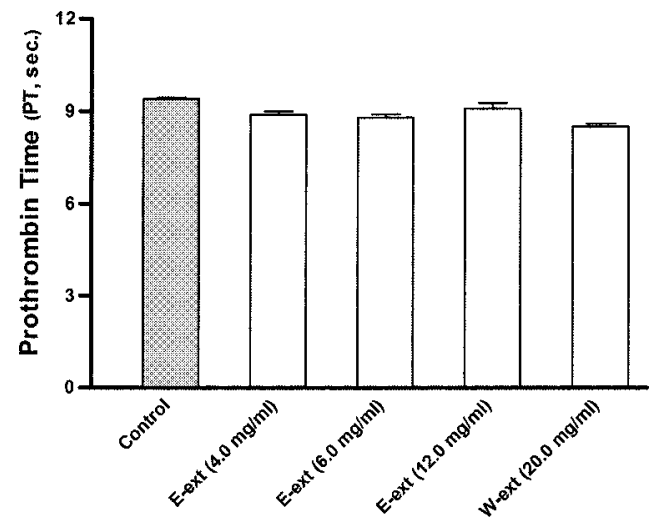

Fig. 2. Effects of BFP on PT Clotting Assay

Each bar is reported as mean (s) \pm S.D. $(n=3-5)$; $* p<0.05, * * p<0.01$, $* * * p<0.001$ significantly different from control, Student's $t$-test. E-ext. and W-ext. mean the BFP $70 \%$ ethanol and aqueous extracts respectively. 
Table 1. Effects of Ingredients from Bak Foong Yun and Heparin on PT, TT, and APTT Assays in Vitro ${ }^{a)}$

\begin{tabular}{|c|c|c|c|}
\hline Sample & TT & PT & APTT \\
\hline Control & $14.7 \pm 0.3$ & $9.4 \pm 0.1$ & $29.6 \pm 1.1$ \\
\hline Astragalus membranaceus & $16.0 \pm 0.4$ & $9.6 \pm 0.5$ & $38.3 \pm 9.1$ \\
\hline Curcuma aeruginosa & $21.3 \pm 0.3^{* * *}$ & $10.8 \pm 0.3^{*}$ & $18.4 \pm 9.1$ \\
\hline Atractylodes macrocephala & $18.1 \pm 1.1$ & $9.0 \pm 0.1$ & $23.2 \pm 8.1$ \\
\hline Ophiopogon japonicus & No test & $9.5 \pm 0.0$ & $24.7 \pm 3.8$ \\
\hline Ligustrum lucidum & $41.9 \pm 1.0^{* * *}$ & $9.6 \pm 0.7$ & $50.4 \pm 5.3^{* *}$ \\
\hline Paeonia albiflora & $17.1 \pm 0.4^{*}$ & $11.6 \pm 0.5$ & $26.7 \pm 4.6$ \\
\hline Phellodendron amurense & $18.1 \pm 0.3$ & $14.6 \pm 0.5^{* * *}$ & $49.5 \pm 8.5^{*}$ \\
\hline Scutellaria baicalensis & $25.8 \pm 1.1 * *$ & $18.2 \pm 0.9^{* *}$ & $244 \pm 7.0 * * *$ \\
\hline Ligusticum chuanxiong & $17.3 \pm 0.3^{*}$ & $11.2 \pm 0.4$ & $17.6 \pm 0.2$ \\
\hline Polygala tenuifolia & $19.9 \pm 0.1$ & $41.5 \pm 1.2 * * *$ & $21.2 \pm 1.2$ \\
\hline Eucommia ulmoides & $20.7 \pm 0.4$ & $10.5 \pm 0.2$ & $17.6 \pm 0.1$ \\
\hline Cyperus rotundus & $16.9 \pm 0.3$ & $12.5 \pm 0.7$ & $26.3 \pm 0.9$ \\
\hline Linum usitatissimum & $20.7 \pm 1.2 *$ & $11.0 \pm 0.1^{*}$ & $51.1 \pm 18.9 * *$ \\
\hline Angelica sinensis & $18.4 \pm 0.2 * * *$ & $10.2 \pm 0.3$ & $21.2 \pm 3.4$ \\
\hline Magnolia officinalis & $23.5 \pm 0.1 * * *$ & $11.0 \pm 0.5$ & $35.0 \pm 16.5$ \\
\hline Leonurus japonicus & $21.9 \pm 0.4 * *$ & $11.1 \pm 0.0^{*}$ & $32.1 \pm 6.2$ \\
\hline Lycopus lucidus & $16.9 \pm 0.5$ & $10.5 \pm 0.2$ & $34.5 \pm 12.6$ \\
\hline Artemisia argyi & $>300^{b)}$ & $43.8 \pm 4.1 * *$ & $>300$ \\
\hline Faeces Trogopterori & $>300$ & $17.2 \pm 3.3$ & $29.0 \pm 1.4$ \\
\hline Corydalis yanhusuo & $20.8 \pm 0.2^{*}$ & $14.2 \pm 0.7 *$ & $98.0 \pm 17.6^{* * *}$ \\
\hline Amygdalus persica & $17.7 \pm 0.6^{* *}$ & $12.0 \pm 0.7 *$ & $23.7 \pm 2.1$ \\
\hline Amomum villosum & $>300$ & $20.4 \pm 2.0^{*}$ & $46.7 \pm 10.1^{*}$ \\
\hline Poria cocos & $30.4 \pm 2.0^{* *}$ & $9.6 \pm 0.1$ & $17.3 \pm 5.2$ \\
\hline Cinnamomum cassia & $>300$ & $10.4 \pm 0.8$ & $38.0 \pm 7.6$ \\
\hline Panax ginseng & $56.0 \pm 4.9^{* *}$ & $16.5 \pm 0.2 * * *$ & $>300$ \\
\hline Cornu Cervi Pantotrichum & $18.7 \pm 0.6$ & $9.6 \pm 0.6$ & $45.7 \pm 10.5$ \\
\hline Heparin $\left.(200 \mu \mathrm{g} / \mathrm{ml})^{c}\right)$ & $>300$ & $21.1 \pm 0.3^{* * *}$ & $>300$ \\
\hline
\end{tabular}

a) PT, TT, and APTT were determined in the presence of the final concentration of $2.0 \mathrm{mg} / \mathrm{ml}$. Data are reported as clotting times (seconds) of plasma (mean \pm S.D., $n=3$ ); $* p<0.05, * * p<0.01, * * * p<0.001$ significantly different from control, Student's $t$-test. $b$ ) No clotting was observed (clotting time $>300 \mathrm{~s}$ ). c) Heparin sodium salt from porcine intestinal, $187 \mathrm{USP}$ units/mg.

cant prolongation of TT ( $p<0.05)$, of which Artemisia argyi, Faeces Trogopterori, Amomum villosum, and Cinnamomum cassia were demonstrated to have potent activities on prolonging TT over $300 \mathrm{~s}$. 11 herbs such as Phellodendron amurense, Polygala tenuifolia, Artemisia argyi, Scutellaria baicalensis, and Panax ginseng showed significant activities prolonging PT. In the APTT assay, BFP extracts as a whole did not show significant effect, but 8 herbs of BFP, such as Ligustrum lucidum, Scutellaria baicalensis, Linum usitatissimum, Artemisia agryi, Corydalis yanhusuo, Amomum villosum, and Panax ginseng showed significant activities prolonging the APTT time $(p<0.05)$. Of these herbs, Artemisia agryi, Amomum villosum, and Panax ginseng prolonged the APTT time over $300 \mathrm{~s}$.

Effects of BFP on Platelet Aggregation The BFP-E-ext showed significant inhibitory activity on platelet aggregation induced by collagen in a doses-dependent manner (Fig. 3). The inhibition percentages were 20,46 , and $74 \%$ at doses of $2.0,4.0$, and $6.0 \mathrm{mg} / \mathrm{ml}$ respectively. The BFP-W-ext exerted less effect on platelet aggregation induced by collagen with inhibitory percentages of 10 and $13 \%$ at doses of 10.0 and $20.0 \mathrm{mg} / \mathrm{ml}$, respectively. For the ADP induced platelet aggregation, the BFP-E-ext exhibited 52\% inhibition at a dose of $6.0 \mathrm{mg} / \mathrm{ml}$, whereas the BFP-W-ext showed $25 \%$ inhibition of platelet aggregation at a dose of $20.0 \mathrm{mg} / \mathrm{ml}$ (Fig. 4). Both BFP ethanol and aqueous extracts were less potent in inhibiting platelet aggregation induced by AA with 4.4 and $14.7 \%$ inhibition at dosages of 4.0 and $6.0 \mathrm{mg} / \mathrm{ml}$, respectively.

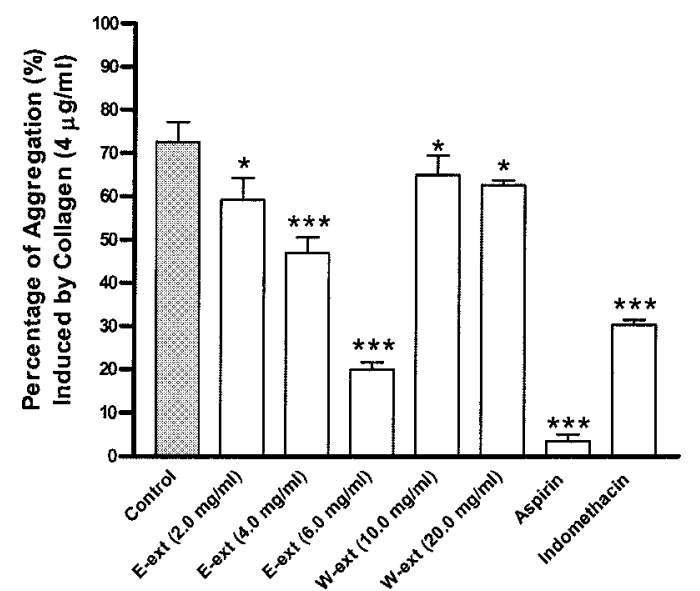

Fig. 3. Effects of BFP on Platelet Aggregation Induced by Collagen

PRP was preincubated with either a test sample, aspirin, indomethacin, or Tris buffer ( $\mathrm{pH} 7.4$ contained $0.3 \% \mathrm{DMSO}$, control) at $37^{\circ} \mathrm{C}$ for $3 \mathrm{~min}$, then collagen $(4 \mu \mathrm{g} / \mathrm{ml})$ was added. Percentages of aggregation are presented as mean \pm S.D. $(n=3) ; * p<0.05$, $* * * p<0.001$, Student's $t$-test. Positive controls, aspirin $(100 \mu \mathrm{g} / \mathrm{ml})$ and indomethacin ( $70 \mu \mathrm{g} / \mathrm{ml})$.

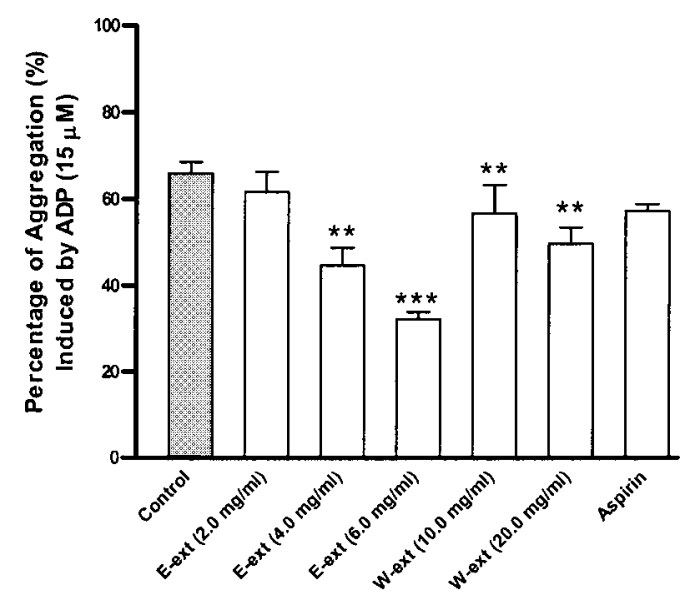

Fig. 4. Effects of BFP on Platelet Aggregation Induced by ADP

PRP was preincubated with either a test sample, aspirin, or Tris buffer ( $\mathrm{pH} 7.4$ contained $0.3 \% \mathrm{DMSO}$, control) at $37^{\circ} \mathrm{C}$ for $3 \mathrm{~min}$, then ADP $(15 \mu \mathrm{M})$ was added. Percentages of aggregation are presented as mean \pm S.D. $(n=3) ; * * p<0.01, * * * p<0.001$, Student's $t$-test. Positive control, aspirin $(100 \mu \mathrm{g} / \mathrm{ml})$.

The effect of individual ingredients of BFP on platelet aggregation was also examined. As shown in Table 2, Angelica sinensis, Magnolia officinalis, Corydalis yanhusuo, Amomum villosum, and Panax ginseng showed significant inhibitory activities of platelet aggregation induced by collagen at a concentration of $1.0 \mathrm{mg} / \mathrm{ml}(p<0.01)$. Of these herbs, Paeonia lactiflora, Corydalis yanhusuo, Amomum villosum, and Panax ginseng also exerted significant inhibitory effects on platelet aggregation induced by ADP $(p<0.01)$. Of all these ingredients, only Ligustum lucidum and Phellodendrom amurense showed strong inhibitory effect on platelet aggregation induced by AA $(p<0.001)$ at a concentration of 1.0 $\mathrm{mg} / \mathrm{ml}$.

Interestingly, 11 herbs of BFP, in contrast to the antiplatelet effect observed with BFP and some of its ingredients, were shown to have effect on induction of platelet aggregation (Fig. 5). Wherein, extracts of Polygala tenuifolia, Eucommia ulmoides, Leonurus japonicus, and Lycopus lucidus strongly induced PRP aggregation with values of 63.0, 
Table 2. Effects of Ingredients of Bak Foong Pills on Platelet Aggregation Induced by Collagen, ADP, and AA in $\mathrm{PRP}^{a}$ )

\begin{tabular}{|c|c|c|c|}
\hline \multirow{2}{*}{ Sample ${ }^{b)}$} & \multicolumn{3}{|c|}{ Aggregation (\%) } \\
\hline & Collagen & ADP & AA \\
\hline Control & $72.7 \pm 4.5$ & $66.0 \pm 2.6$ & $69.3 \pm 1.5$ \\
\hline Ligustum lucidum & $47.4 \pm 4.1^{*}$ & $51.3 \pm 3.4^{*}$ & $60.7 \pm 0.9 * * *$ \\
\hline Poria cocos & $69.0 \pm 0.8$ & $57.3 \pm 2.6$ & $68.0 \pm 2.2$ \\
\hline Paeonia lactiflora & $60.0 \pm 6.4^{*}$ & $60.3 \pm 2.5 * *$ & $61.7 \pm 2.1^{*}$ \\
\hline Phellodendron amurense & $53.0 \pm 5.0 *$ & $29.3 \pm 9.2 *$ & $17.7 \pm 2.9 * * *$ \\
\hline Linum usitatissimum & $47.3 \pm 1.9 *$ & $60.3 \pm 4.0^{*}$ & $57.5 \pm 1.5^{*}$ \\
\hline Angelica sinensis & $22.7 \pm 4.5^{* *}$ & $47.7 \pm 6.0^{*}$ & $58.5 \pm 5.0 *$ \\
\hline Magnolia officinalis & $39.7 \pm 6.8 * *$ & $52.0 \pm 1.6^{*}$ & $59.5 \pm 4.9 *$ \\
\hline Artemisia argyi & $76.0 \pm 1.6$ & $66.0 \pm 1.4$ & $68.3 \pm 2.5$ \\
\hline Faeces Trogopteroti & $65.3 \pm 6.9$ & $57.7 \pm 6.2$ & $66.7 \pm 3.4$ \\
\hline Corydalis yuanhusuo & $41.0 \pm 4.3 * *$ & $39.3 \pm 3.9 * * *$ & $41.3 \pm 5.2 * *$ \\
\hline Cyperus rotundus & $67.3 \pm 1.7$ & $52.7 \pm 5.8$ & $61.0 \pm 1.0$ \\
\hline Amomum villosum & $45.7 \pm 6.1 * *$ & $55.0 \pm 2.4 * * *$ & $50.0 \pm 3.3 * *$ \\
\hline Atractylodes macrocephala & $70.5 \pm 3.0$ & $52.7 \pm 3.4$ & $64.3 \pm 2.9$ \\
\hline Cinnamomum cassia & $74.0 \pm 1.4$ & $41.7 \pm 4.2 *$ & $61.0 \pm 1.0$ \\
\hline Panax ginseng & $51.0 \pm 3.6^{* * *}$ & $53.7 \pm 4.8^{* *}$ & $55.3 \pm 4.0 *$ \\
\hline Aspirin & $3.5 \pm 1.5^{* * *}$ & $57.3 \pm 1.5^{* *}$ & $1.3 \pm 0.5^{* * *}$ \\
\hline Indomethacin & $30.3 \pm 2.1 * * *$ & $40.0 \pm 9.0 *$ & $1.7 \pm 0.6^{* * *}$ \\
\hline
\end{tabular}

a) PRP was preincubated with either a test sample, aspirin, indomethacin, or Tris buffer ( $\mathrm{pH} 7.4$ contained $0.3 \% \mathrm{DMSO}$, control) at $37^{\circ} \mathrm{C}$ for $3 \mathrm{~min}$, then collagen $(4 \mu \mathrm{g} / \mathrm{ml})$, ADP $(15 \mu \mathrm{M})$, AA $(15 \mu \mathrm{M})$ was added. Percentages of aggregation are presented as mean \pm S.D. $(n=3) ; * p<0.05, * * p<0.01, * * * p<0.001$, Student's $t$-test. b) Sample dose was $1.0 \mathrm{mg} / \mathrm{ml}$; aspirin $(100 \mu \mathrm{g} / \mathrm{ml})$, and indomethacin $(70 \mu \mathrm{g} / \mathrm{ml})$.

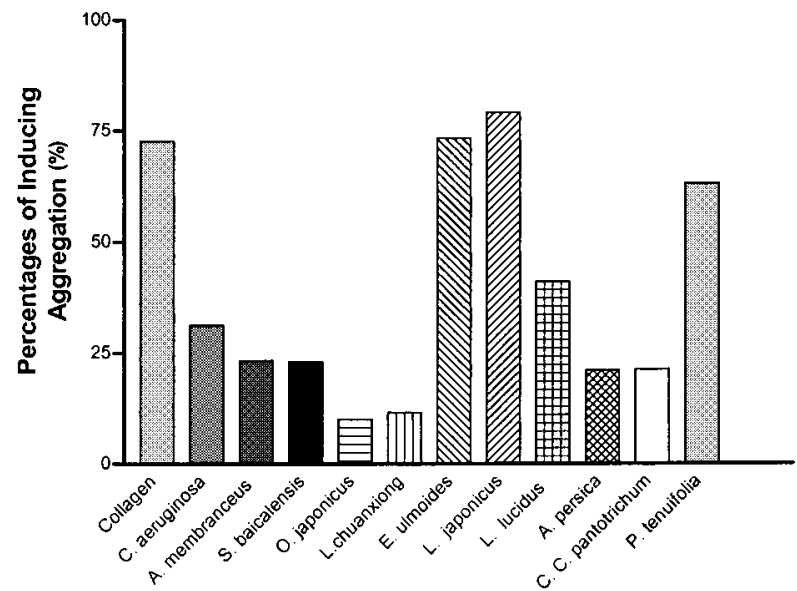

Fig. 5. Effects of Inducing Platelet Aggregation by Ingredients of BFP

PRP was preincubated at $37^{\circ} \mathrm{C}$ for $3 \mathrm{~min}$, then collagen $(4 \mu \mathrm{g} / \mathrm{ml})$ and samples $(1.0 \mathrm{mg} / \mathrm{ml})$ were added. Bars showed percentages of inducing platelet aggregation by samples and collagen $(n=3$, averages). The percentage of aggregation was determined in $10 \mathrm{~min}$.

73.3, 79.0 and $41.0 \%$ (Collagen as the control), respectively, at a concentration of $1.0 \mathrm{mg} / \mathrm{ml}$.

In Vivo Bleeding Time The anticoagulation and antiplatelet effects of BFP were further demonstrated by examining the bleeding time in vivo, a process depending on both blood coagulation and platelet activity. Bleeding times were measured in mice fed with the BFP-E-ext for 2 weeks before amputating $1 \mathrm{~cm}$ of the tail tip. Results showed that BFP significantly prolonged the bleeding times at doses of 2,4 , and $6 \mathrm{~g} / \mathrm{kg}$, respectively (Fig. 6). A bell-shaped dose response was observed indicating reversed effect on the bleeding time at higher concentrations.

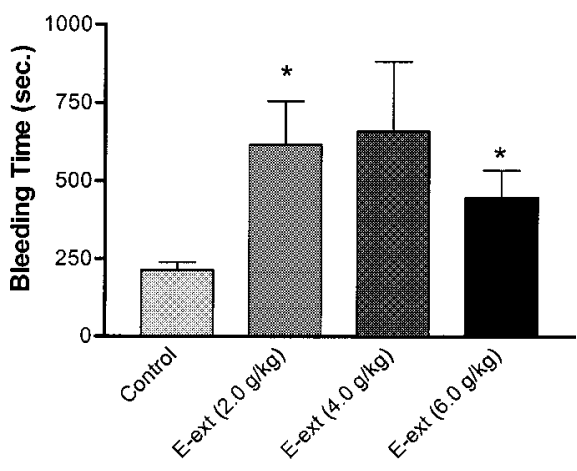

Fig. 6. Bleeding Times in ICR Mice

The ICR mice fed with BFP for 2 weeks before the bleeding time experiment. Each bar is reported as mean (s) \pm S.D. $(n=9-10) ; * p<0.05$, significantly different from control, Student's $t$-test. E-ext. means the BFP $70 \%$ ethanol.

\section{DISCUSSION}

BFP is a remedy well known for its beneficial effects on gynecological disorders with clinical indications for its effect on overall body function including the cardiac vascular system. However, the mechanisms underlying the beneficial effects of BFP are far from understood. The present study was the first attempt to investigate the effect of BFP on blood coagulation and platelet aggregation.

Various in vitro coagulation assays such as PT, TT, and APTT, can be used to study the extrinsic and intrinsic coagulation cascade. ${ }^{14)}$ Blood coagulation may be initiated through either the intrinsic pathway, where all of the protein components are present in blood, or the extrinsic pathway, where the cell-membrane protein tissue factor plays a critical role. ${ }^{15)}$ The PT and TT assays involve procedures that initiate the extrinsic pathway, whereas, the APTT assay involves procedures that initiate the intrinsic pathway. ${ }^{15,16)}$ In our study, the effect of BFP on anticoagulation was evaluated using PT, TT, and APTT assays. It was seen that the BFP-E-ext significantly prolonged the TT in vitro in a dose-dependent manner, whereas, the BFP-W-ext showed weaker activity on prolonging thrombin time. However, both the BFP ethanol and aqueous extract were not able to prolong clotting activities in PT and APTT assays. Suggesting that BFP exhibits anticoagulant effect mainly through the extrinsic pathway.

PT, TT, and APTT assays involve procedures that initiate the extrinsic and/or intrinsic pathways by activation of a specific step of the cascade. Because of their specificity in initiating the cascade, the assays provide a systematic approach to evaluate the inhibition by active extracts and help in identifying the complexes in the extrinsic and/or intrinsic pathways where they inhibit. Activating the cascade upstream from the inhibited step will result in elevated clotting times of the plasma, while activating downstream of the inhibited step will not affect the clotting time. ${ }^{14)}$ The BFP significantly prolonged the clotting time of plasma as measured with the TT assay, but no significant effects were observed on the PT and APTT times. These results indicate that BFP directly inhibits the thrombin enzyme, the key enzyme in the blood coagulation cascade. The above results strongly suggested that anticoagulant effects of BFP, as a whole, are mediated by a mechanism directly blocking thrombin during blood coagulation. 
Analysis of the BFP preparation indicates that 17 of the 26 ingredients in BFP have significant effects in prolonging the TT, while 11 herbs show significant effects on the PT and 8 herbs significantly prolong APTT, indicating that the majority of individual ingredients of BFP have overall anticoagulant effect either through intrinsic and/or extrinsic pathways.

Naturally occurring herbs have been extensively screened for their potentials as new anticoagulant agents. ${ }^{4,17,18)}$ Our present screening of the ingredients of BFP also indicates possible new anticoagulant agents, such as Faece trogopterora, Amomum villisum, and Cinnamomum assia, which were shown to have selective prolongation of the thrombin time over $300 \mathrm{~s}$ without clotting of the plasma indicating a direct effect on thrombin. Because the central role of thrombin in homeostasis and thrombosis has long been appreciated from a scientific and practical point of view, particular attention has been given to seeking thrombin-direct inhibitors in recent researches. ${ }^{19,20)}$ Our findings suggest further investigation on those candidate herbs directly inhibiting thrombin. It is interesting to note that Artemisia argyi exhibited potent anti-clotting activities in both TT and APTT assays while Panax ginseng showed selective anticoagulant activity through intrinsic pathway by significantly prolonging the APTT over $300 \mathrm{~s}$. A combination of these herbs with potent anticoagulation effects acting on both intrinsic and extrinsic pathways may be proved to be effective therapeutic agents for preventing thrombosis or stroke.

The present study has also investigated the antiplatelet effects of BFP, both the ethanol and aqueous extracts as well as extracts of individual herbs, by observing inhibitory activities of platelet aggregation induced by collagen, AA, or ADP. Results showed that BFP significantly inhibited platelet aggregation induced by collagen and ADP, but with rather minimal inhibitory effect on platelet aggregation induced by AA. Again, the effect of ethanol extract was much more pronounced than that of the aqueous extract. It should be noted that while aspirin $(100 \mu \mathrm{g} / \mathrm{ml})$, a well-known antiplatelet agent, was most effective in reducing collagen and AA-induced platelet aggregation, its effect on ADP-induced platelet aggregation was rather minimal, significantly less than that produced by BFP-E-ext. This suggests that the potential of BFP as antiplatelet agent with alternative mechanism may be further explored.

Possible actions of BFP on platelet aggregation involving different mechanisms are shown to be complicated as evident by the results obtained from the studies of the 26 ingredients of BFP. Some of the ingredients examined have been reported to have antiplatelet activities. For example, active ingredients of Magnolis officinalis, ${ }^{22)}$ Corydalis yanhusuo, ${ }^{21)}$ and Panax ginseng ${ }^{23,24)}$ have been shown to exert their antiplatelet effect through a mechanism inhibiting thromboxane formation. Magnolia officinalis and Corydalis yuanhusuo have also been shown to exert antiplatelet action through the decrease of intracellular calcium concentration. ${ }^{21)}$ or inhibition of intracellular calcium mobilization. ${ }^{22}$ ) Our preliminary studies indicate that the antiplatelet effect of some of the ingredients of BFP may be mediated by nitric oxide, a molecule with increasingly recognized role in platelet aggregation. $^{25)}$

It is interesting to note that 11 herbs in BFP are able to induce platelet aggregation in rabbit PRP (Fig. 5). Wherein, extracts of Polygala tenuifolia, Eucommia ulmoides, Leonurus japonicus, and Lycopus lucidus strongly induced PRP aggregation to the same degree as collagen. In the present time, little is known for the action mechanism of these ingredients in inducing platelet aggregation. However, it is expected that they may play a very important role in counter-acting the antiplatelet action of some of the BFP ingredients and preventing over bleeding for women taking BFP, especially during menstruation.

The anticoagulant and antiplatelet effects of BFP could be reflected collectively by the bleeding time study conducted in mice 2 weeks after treatment with BFP. The bleeding time in BFP-treated animals, for all concentrations of BFP-E-ext examined, was significantly increased (Fig. 6). This action could attribute to the anticoagulant and/or antiplatelet effects of BFP ingredients. It is interesting to note the bell-shaped of the dose response of BFP on the bleeding time which suggests reversed effect on blood coagulation at higher BFP concentrations used. The BFP ingredients capable of inducing platelet aggregation may be responsible for the reversed effect of high concentration of BFP on blood coagulation. Considering that BFP is not a remedy specifically designed for targeting the cardiovascular system, the bell-shaped response of BFP effect thus seems to be beneficial for prevention of unnecessary bleeding for women using BFP.

The BFP effect on blood coagulation in vivo may also attribute to its effect on levels of estrogen, in addition to its direct anticoagulation and antiplatelet effect, since estrogen replacement has been reported to suppress function of thrombin-stimulated platelets. ${ }^{26}$ and has also been reported to decrease intrinsic coagulation factors. ${ }^{27)}$ This notion could be supported by the well-known use of BFP for treating gynecological disorders through its estrogenic action. Changes in estrogen levels (unpublished data) and estrogenic effects of BFP on the female reproductive tract have also been observed in BFP-treated mice. ${ }^{8)}$ Taken together, BFP exhibits significant anticoagulation and antiplatelet aggregation activities, either due to direct effects of its active ingredients or through its action on levels of estrogen. The effects of BFP on the blood observed in the present study, in addition to that on estrogen levels, contributes to the beneficial effects of BFP on overall body functions. The combined effects of BFP appear to be particularly beneficial for postmenopausal women who have reduced levels of estrogen and are known to have high prevalence of coronary heart disease ${ }^{9)}$ as well as other cardiovascular diseases.

Acknowledgement This work was supported by the Innovation Technology Fund of Hong Kong.

\section{REFERENCES}

1) Davie E. W., Ratnoff O. D., Science, 145, 1310-1312 (1964).

2) Kimball S. D., Blood Coag. Fibrinol., 6, 511-519 (1995).

3) Beretz A., Cazenave T. P., Planta Med., 57 (Suppl. 1), 68-72 (1991).

4) Okuyama T., Narui T., Kurata H., Wang J. D., Hashimoto T., J. Med. Pharm. Soc. Wakan-Yaku, 5, 167-178 (1988).

5) Dong H., Chen S. X., Kini R. M., Xu H. X., J. Nat. Prod., 61, 13561360 (1998)

6) Chung M. I., Gan K. H., Lin C. N., Ko F. N., Teng C. M., J. Nat. Prod., 56, 929-934 (1993).

7) Zhu J. X., Chan Y. M., Tsang L. L., Chan L. N., Zhou Q., Zhou C. X., Chan H. C., Jpn. J. Physiol., 52, 129-134 (2002). 
8) Rowlands D. K., Tsang L. L., Cui Y. G., Chung Y. W., Chan Y. M., Chan L. N., Liu C. Q., James T., Chan H. C., Cell Biol. Inter, 25, 1033-1035 (2001).

9) Nakano Y., Oshima T., Matsuura H., Kajiyama G., Kambe M., Arterioscler Thromb. Vasc. Biol., 18, 961-967 (1998).

10) Barrett-Conner E., Bush T. L., JAMA., 265, 1861-1867 (1991).

11) Kettner C., Mersinger L., Knabb R. J., J. Biol. Chem., 30, $18289-$ 18297 (1990).

12) Offermanns S., Toombs C. F., Hu Y. H., Simon M. I., Nature (London), 389, 183-186 (1997).

13) Born G. V. R., J. Physiol. (London), 126, 67-71 (1977).

14) Stefansson S., Kini R. M., Evans H. J., Thromb. Res., 55, 482-491 (1989).

15) Furie B., Furie B. C., Cell, 53, 505-518 (1988).

16) Akoum A., Josefonvicz J., Sigot M., Thromb. Res., 60, 9-18 (1990).

17) Wan D. D., Girard T. J., Kasten T. P., Lachance R. M., Miller-Wideman M. A., Durley R. C., J. Nat. Prod., 61, 1352-1355 (1998).

18) O’Neill M. J., Lewis J. A., Nobile H. M., Holland S., Mansat C., Far- thing J. E., Foster G., Nobile D., Lane S. J., Sidebottom P. J., Lyum S. M., Hayes M. V., Dix C. J., J. Nat. Prod., 61, 1328-1331 (1998).

19) Fenton J. W., Ann. N.Y. Acad. Sci., 485, 5-16 (1986).

20) Fenton J. W., Blood Coag. Fibrinol., 2, 69-75 (1991).

21) Ko F. N., Wu T. S., Lu S. T., Wu Y. C., Huang T. F., Teng C. M., Thromb. Res., 56, 289-298 (1989).

22) Teng C. M., Chen C. C., Ko F. N., Lee L. G., Huang T. F., Chen Y. P., Hsu H. Y., Thromb. Res., 50, 757-765 (1988).

23) Teng C. M., Kuo S. C., Ko F. N., Lee J. C., Lee L. G., Chen S. C., Huang T. F., Biochim. Biophys. Acta, 990, 315-320 (1989).

24) Kuo S. C., Teng C. M., Lee J. C., Ko F. N., Chen S. C., Wu T. S., Planta Med., 56, 164-167 (1990).

25) Loscalzo J., Circulation Research, 27, 756-762 (2001).

26) Nakano Y., Oshima T., Ozono R., Ueda A., Oue Y., Matsuura H., Sanada M., Ohama K., Chayama K., Kambe M., Cardiovascular Research, 53, 634-641 (2002).

27) Demirol A., Baykal C., Kirazli S., Ayhan A., Menopause, 8, 135-140 (2001). 